Geometry \& Topology

Volume 4 (2000) 171-178

Published: 19 July 2000

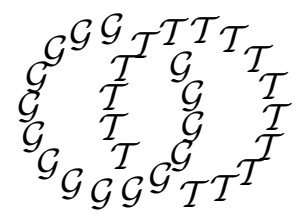

\title{
On the geometric boundaries of hyperbolic 4-manifolds
}

\author{
D D LONG \\ A W REID \\ Department of Mathematics, University of California \\ Santa Barbara, CA 93106, USA \\ and \\ Department of Mathematics, University of Texas \\ Austin, TX 78712, USA
}

Email: long@math.ucsb.edu and areid@math.utexas.edu

\begin{abstract}
We provide, for hyperbolic and flat 3-manifolds, obstructions to bounding hyperbolic 4-manifolds, thus resolving in the negative a question of Farrell and Zdravkovska.
\end{abstract}

AMS Classification numbers Primary: 57R90

Secondary: $57 \mathrm{M} 50$

Keywords: Hyperbolic 3-manifold, flat manifold, totally geodesic, $\eta$-invariant

Proposed: Walter Neumann

Seconded: Jean-Pierre Otal, Robion Kirby
Received: 18 June 2000

Accepted: 19 July 2000

Copyright Geometry and Topology 


\section{Introduction}

It is a classical result of Rohlin that the bordism group of closed orientable 3 -manifolds is zero, so that every such $M^{3}$ can be identified with $\partial W^{4}$ for some appropriate compact $W^{4}$.

This paper deals with two geometric incarnations of this situation. The first, motivated in part by considerations in physics, (see [7], [16] and [20]), asks whether a closed orientable hyperbolic 3 -manifold can be the totally geodesic boundary of a compact complete hyperbolic 4-manifold. The second, motivated by a theorem of Hamrick and Royster [10] (which shows that that in every dimension a flat $k$-manifold is nullbordant), concerns the question of whether every flat 3-manifold is, up to homeomorphism, a cusp cross-section of a complete finite volume 1-cusped hyperbolic 4-manifold.

We introduce the following notation. If a hyperbolic 3 -manifold $M$ is the totally geodesic boundary of a hyperbolic 4-manifold $W$, we say $M$ bounds geometrically. Also recall that the $\eta$-invariant of $M$, denoted $\eta(M)$ is defined as $\eta(0)$ where $\eta(s)$ is formed from a signed collection of eigenvalues of a certain first order self adjoint operator on $M$.

In this note we show:

Theorem 1.1 If a closed hyperbolic $M^{3}$ bounds geometrically, then $\eta(M) \in$ Z.

It has been shown in [13], that as we run over surgeries on a hyperbolic knot in $S^{3}$ the $\eta$-invariant takes on a dense set of values in $\mathbf{R}$, so we deduce:

Corollary 1.2 There are closed hyperbolic 3-manifolds which do not bound geometrically.

These are the first known examples of hyperbolic 3-manifolds, that while they are nullbordant, are not nullbordant in this geometrical sense; see question 4 of $[20]$.

In the flat case, we show a similar theorem:

Theorem 1.3 If a closed flat $M^{3}$ is the cusp cross-section of a complete finite volume one-cusped hyperbolic $W^{4}$, then $\eta(M) \in \mathbf{Z}$. 
In [14] (see also [12]) it is shown that the homemorphism type of every flat 3-manifold appears as a cusp cross-section of a complete finite volume cusped hyperbolic 4-manifold, possibly with several cusps. Using some calculations of [15], one can show that there are flat 3 -manifolds with nonintegral $\eta$-invariant (see the calculations below) so that we deduce:

Corollary 1.4 There are closed flat 3-manifolds which are not homeomorphic to the cusp cross-section of any complete finite volume one-cusped hyperbolic 4-manifold.

The proof of Theorems 1.1 and 1.3 use a celebrated formula of Atiyah-PatodiSinger, but less refined geometrical considerations still give information:

Theorem 1.5 Let $M(n)$ be an infinite sequence of distinct closed hyperbolic 3 -manifolds. Suppose that, for each $n, M(n)$ is the totally geodesic boundary of a hyperbolic 4-manifold $W(n)$.

Then $\chi(W(n)) \rightarrow \infty$ as $n \rightarrow \infty$, where $\chi$ denotes Euler characteristic.

Using the above and a result of Gordon [6] we deduce:

Corollary 1.6 There are hyperbolic integral homology 3-spheres that bound rationally acyclic (in fact contractible) 4-manifolds but cannot geometrically bound any rationally acyclic hyperbolic manifold.

\section{Proofs}

The starting point of this work is the following theorem of Atiyah, Patodi and Singer:

Theorem 2.1 (See [1], [2]) Let $W$ be a compact oriented Riemannian 4manifold with boundary $M$ and assume that near $M$, the metric is isometric to a product.

Then

$$
\operatorname{sign}(W)=\frac{1}{3} \int_{W} p_{1}-\eta(M)
$$


We briefly explain the terminology of the theorem. The left hand side is the signature of the nondegenerate symmetric form on the image of $H^{2}(W, M ; \mathbf{Z})$ in $H^{2}(W ; \mathbf{Z})$ induced via the cup product, $p_{1}$ is the differential 4 -form representing the first Pontryagin class; in [2] this is defined as $(2 \pi)^{-2} \operatorname{tr}(R \wedge R)$, where $R$ is the curvature matrix.

We wish to apply this theorem in the contexts provided by 1.1 and 1.3 and there is a technical point that in neither case is the hyperbolic metric going to be a product near the boundary. However, as pointed out in [2] page 61, (see also [8]) the correction term necessary if the metric is not a product near the boundary is expressible in terms of an integral involving the curvature and the second fundamental form $\theta$; this expression is given explicitly in [5] pages $348-9$ as

$$
\int_{\partial W} \operatorname{tr}(\theta \wedge R)
$$

We do not need to appeal to the details of this formula, for the two cases that interest us we will argue that the correction term must be zero.

We now complete the proof of Theorem 1.1. Suppose that $M$ is hyperbolic and bounds geometrically the hyperbolic 4 -manifold $W$. Since we are assuming that the boundary is totally geodesic, it follows that the second fundamental form is zero (see [4] Theorem 2 page 194) and the formula of 2.1 holds without correction. Now hyperbolic manifolds are conformally flat, (one simply notes that the standard injection $\mathrm{SO}_{0}(n, 1) \rightarrow \mathrm{SO}_{0}(n+1,1)$ preserving a codimension one totally geodesic subspace of $\mathbf{H}^{n+1}$ gives a conformal action on $S^{n}$ ) and since the Pontryagin form is a conformal invariant (see [2]), it must be identically zero. Then the formula reduces to

$$
\operatorname{sign}(W)=-\eta(M)
$$

so that $\eta(M)$ is an integer as required.

The proof of Theorem 1.3 is similar, but we need to argue a little differently to compute the correction since in this case the second fundamental form is not zero.

Truncate the manifold $W$ with a small horoball, of Euclidean height $k$ say. This gives a compact 4-manifold which we denote by $W(k)$. This manifold has flat boundary and we may write the Index formula in this case as:

$$
\operatorname{sign}(W)-\frac{1}{3} \int_{W} p_{1}+\eta(M(k))=\operatorname{correction}(k),
$$

\section{Geometry and Topology, Volume 4 (2000)}


where $M(k)$ is isometric to the flat manifold $M$. As above there is no contribution from the integral term on the left hand side. Moreover, the $\eta$-invariant of a flat 3-manifold is independent of choice of flat metric ([15] and see the calculation of $\eta(M)$ below) so that the left hand side does not depend on $k$.

Now from above, the term correction $(k)$ is formed by integrating over the boundary a continuous locally computable quantity which is isometry invariant, so that the correction is bounded above by $C \cdot \operatorname{volume}(M(k))$ where $C$ is a constant independent of $k$.

Since volume $(M(k))$ tends to 0 as the horoballs get smaller, it follows that the correction term must be zero.

Thus $\operatorname{sign}(W)=-\eta(M)$ is integral as required.

Remarks (1) In the closed case, Theorem 2.1 (which reduces to the Hirzebruch signature formula in this case) and the same $\operatorname{argument} \operatorname{shows} \operatorname{sign}(W)=0$ for a closed hyperbolic $4 k$-manifold.

(2) All known examples of hyperbolic 4-manifolds of finite volume contain immersed totally geodesic hyperbolic 3 -manifolds which give embedded totally geodesic hyperbolic $3-$ manifolds in finite covers. However, until recently, no single example of a hyperbolic 3-manifold that did bound geometrically was known; the first example was given [16].

(3) The smallest known hyperbolic 3-manifold with $\eta(M) \in \mathbf{Z}$ is the manifold identified as Vol3, with volume that of the regular ideal simplex in $\mathbf{H}^{3}$; one description of this manifold being $(3,-2),(6,-1)$ surgery in the Whitehead link complement (see [13]). In fact $\eta(\operatorname{Vol} 3)=0$ (see [13]). Note the volume of the 3-manifold constructed in [16] that does bound geometrically is of the order of 200 . It has $\eta$-invariant 0 .

Proof of Theorem 1.5 In even dimensions, hyperbolic volumes are basically the same as Euler characteristics [9]. In dimension 4, the exact statement is that if $M$ is a complete hyperbolic 4-manifold of finite volume, then

$$
\operatorname{Vol}(M)=\frac{4 \pi^{2}}{3} \chi(M)
$$

Furthermore by results of Wang [19], there at most a finite number of isometry classes of finite volume hyperbolic 4-manifolds of given volume. Thus for a given constant $C$, there are only a finite number of distinct closed hyperbolic 4 -manifolds whose Euler characteristic is $<C$. 
In the notation of 1.5, suppose that there were an infinite sequence with bounded Euler characteristics and let $D(W(n))$ be the closed hyperbolic 4-manifolds formed by doubling along the boundary. These have bounded Euler characteristics so we may pass to a subsequence so that the doubled manifolds are all homeomorphic, hence isometric by Mostow rigidity.

This is already a contradiction to a theorem of Basmajian [3] if the volumes of the the $M(n)$ are unbounded. If the volumes of the $M(n)$ remain bounded, then since they are all isometrically embedded in a single closed hyperbolic 4-manifold, there is a global lower bound on the injectivity radius in the sequence and this is also a contradiction, since there are only a finite number of distinct closed hyperbolic 3-manifolds with injectivity radius bounded below and volume bounded above, [18].

It is observed in [6] that $1 / n$ surgery on a slice knot bounds a contractible manifold; 1.5 shows that only finitely many such manifolds could geometrically bound anything rationally acyclic.

\section{Calculation of $\eta(M)$ for flat 3-manifolds}

In [15], formulae for the $\eta$-invariants of Seifert fibered 3-manifolds were developed. In particular for a flat 3-manifold $M$, it is shown that $\eta(M)$ depends only on the topology of $M$ and is independent of the flat metric. From the theory of flat Seifert fibered manifolds, there is a unique orientable flat 3-manifold with base for the Seifert fibration $S^{2}$ and Seifert invariants $(2,1),(3,-1),(6,-1)$.

From [15], $\eta(M)$ is given by:

$$
\begin{aligned}
\eta(M)=4\left(\frac{1}{8} \cot ^{2} \pi / 2+\frac{1}{12}\right. & \left(-\cot ^{2} \pi / 3+\cot ^{2} 2 \pi / 3\right) \\
& +\frac{1}{24} \Sigma_{k=1}^{5} \cot (-k \pi / 6) \cot (k \pi / 6) .
\end{aligned}
$$

This calculation gives $\eta(M)=-4 / 3$, as required in Corollary 1.4.

On repeating this calculation for the six other orientable flat 3-manifolds, the $\eta$-invariant is integral except for the unique Seifert manifold with base $S^{2}$ and Seifert invariants $(3,2),(3,-1),(3,-1)$ for which the answer is $-2 / 3$.

\section{Higher dimensional manifolds}

Although the focus of this note has been motivated by the considerations coming from 4-manifolds, many of our results continue to hold to obstruct $4 n-1$ 
manifolds as the totally geodesic or cusp cross-sections of $4 n$-manifolds. The Atiyah-Patodi-Singer theorem holds verbatim as does the formula for the correction term. It follows that geometric bounding in the totally geodesic case is obstructed exactly as above by the $\eta$-invariant. However, in contrast to the low dimensional case, we do not know how to compute any higher dimensional $\eta$-invariants. There is no analogue of the results of [13] in dimensions $\geq 4$.

The case of a cusp cross-section also works similarly, but we need to make the additional observation (see [2]) that scaling the metric does not change the $\eta-$ invariant. Since the flat metrics on horoball cross-sections at different heights differ only by scaling, the term correction $(k)$ continues to be independent of $k$ and the geometrical argument above still works to show correction $(k)=0$. We do not have explicit flat $4 n-1$-manifolds $(n>1)$ for which the $\eta$-invariant is non-integral, but it seems likely that such do exist.

Acknowledgements The authors are indebted to Xianhze Dai for several enormously helpful and stimulating conversations. Discussions with CMcA Gordon, and email correspondence with W Neumann were also very informative.

The first author was partially supported during this work by the NSF. The second author was partially supported by the NSF, the Alfred P Sloan Foundation and a grant from the Texas Advanced Research Program.

\section{References}

[1] M F Atiyah, V K Patodi, I M Singer, Spectral asymmetry and Riemannian geometry, Bull. LMS, 5 (1973) 229-234

[2] M F Atiyah, V K Patodi, I M Singer, Spectral asymmetry and Riemannian geometry, I, Math. Proc. Camb. Phil. Soc. 77 (1975) 43-69

[3] A Basmajian, Tubular neighbourhoods of totally geodesic hypersurfaces in hyperbolic manifolds, Inventiones Math. 117 (1994) 207-225

[4] R L Bishop, R J Crittenden, Geometry of Manifolds, Academic Press, New York (1964)

[5] T Eguchi, P B Gilkey, A J Hanson, Gravitation, gauge theories and differential geometry, Physics Reports, 66 (1980) 213-393

[6] CMcA Gordon, Knots, homology spheres and contractible 4-manifolds, Topology, 14 (1975) 151-172

[7] G W Gibbons, Tunneling with a negative cosmological constant, Nuclear Phys. B, 472 (1996) 683-708 
[8] P B Gilkey, The boundary integrand in the formula for the signature and Euler characteristic of a Riemannian manifold with boundary, Adv. Math. 15 (1975) 344-360

[9] M Gromov, Volume and bounded cohomology, Publ. IHES, 56 (1982) 5-99

[10] G Hamrick, D Royster, Flat Riemannian manifolds are boundaries, Invent. Math. 66 (1982) 405-413

[11] F T Farrell, S Zdravkovska, Do almost flat manifolds bound, Michigan J. Math. 30 (1983) 199-208

[12] D D Long, A W Reid, All flat manifolds are cusps of hyperbolic orbifolds, preprint

[13] R Meyerhoff, W D Neumann, An asymptotic formula for the eta invariant for hyperbolic 3-manifolds, Comment. Helv. Math. 67 (1992) 28-46

[14] B E Nimershiem, All flat three-manifolds appear as cusps of hyperbolic fourmanifolds, Topology and its Appl. 90 (1998) 109-133

[15] M Ouyang, Geometric invariants for Seifert fibred 3-manifolds, Trans. AMS, 346 (1994) 641-659

[16] J G Ratcliffe, S T Tschantz, Gravitational instantons of constant curvature, Classical and Quantum Gravity, 15 (1998) 2613-2627

[17] J G Ratcliffe, S T Tschantz, The volume spectrum of hyperbolic 4-manifolds, Experimental J. Math. 9 (2000) 101-125

[18] W P Thurston, Three-Dimensional Geometry and Topology, Volume 1, Princeton University Press (1997)

[19] H C Wang, Topics in totally discontinuous groups, from; "Symmetric Spaces", W M Boothby and GL Weiss editors, Pure and Appl. Math 8, Marcel Dekker (1972) 459-487

[20] J Weeks, Topological questions in cosmology, to appear in the Proceedings of the Oklahoma conference on computational topology 